\title{
Cost effectiveness of outpatient treatment for febrile neutropaenia in adult cancer patients
}

\author{
O Teuffel', E Amir ${ }^{2}$, S Alibhai ${ }^{3}$, J Beyene ${ }^{4}$ and L Sung*,' \\ 'Division of Haematology/Oncology, Child Health Evaluative Sciences, The Hospital for Sick Children, 555 University Avenue, Toronto, Ontario, Canada \\ M6G IX8; '2Division of Medical Oncology, Princess Margaret Hospital, 610 University Avenue, Toronto, Ontario, Canada M5G 2M9; ${ }^{3}$ Department of \\ Medicine, University Health Network, 200 Elizabeth Street, Toronto, Ontario, Canada M5G 2C4; ${ }^{4}$ Dalla Lana School of Public Health, University of \\ Toronto, 27 King's College Circle, Toronto, Ontario, Canada M5S IAI
}

BACKGROUND: There is uncertainty whether low-risk episodes of febrile neutropaenia (FN) in adult cancer patients are best managed in the in- or outpatient setting.

METHODS: A Monte Carlo cost-utility model was created to compare four treatment strategies for low-risk FN: (I) treatment in hospital with intravenous antibiotics (HosplV); (2) early discharge after $48 \mathrm{~h}$ in-patient observation, followed by oral outpatient treatment (EarlyDC); (3) outpatient management with IV antibiotics (HomelV); and (4) outpatient management with oral antibiotics (HomePO). The model used a health-care payer perspective and a time horizon of one FN episode. Outcome measures were quality-adjusted FN episodes (QAFNE), costs (Canadian dollars) and incremental cost-effectiveness ratios (ICER). Parameter uncertainty was assessed with probabilistic sensitivity analyses.

RESULTS: HomePO was cost saving (\$3470 vs \$4183), but less effective (0.65 QAFNE vs 0.72 QAFNE) than HomelV. The corresponding ICER was \$I I86 per QAFNE. Both EarlyDC (\$6I I5; 0.66 QAFNE) and HospIV (\$I3 557; 0.62 QAFNE) were dominated strategies. At a willingness-to-pay (WTP) threshold of $\$ 4000$ per QAFNE, HomePO and HomelV were cost effective in 54 and $38 \%$ of simulations, respectively.

INTERPRETATION: For adult cancer patients with an episode of low-risk FN, treatment in hospital is more expensive and less effective than outpatient strategies.

British Journal of Cancer (201 I) 104, 1377-1383. doi:10.1038/bjc.2011.101 www.bjcancer.com

Published online 5 April 201।

(C) 201। Cancer Research UK

Keywords: cost effectiveness; fever; neutropaenia; ambulatory care; in-patients

Despite recent advances in infection prevention, febrile neutropaenia (FN) remains a frequent complication of chemotherapy in adult cancer patients (Renwick et al, 2009). For many decades, owing to the potential for life-threatening sepsis, the standard treatment of FN had been in-patient management with broadspectrum intravenous (IV) antibiotics for all patients (Hughes et al, 2002). It is now well recognised, however, that adult patients with FN are a heterogeneous population, with only a small proportion developing serious medical complications (Klastersky et al, 2000). Consequently, current national and international guidelines have endorsed less aggressive empiric antibiotic strategies, including outpatient and/or oral antibiotic regimens, for adult cancer patients with low-risk FN (Tamura, 2005; Segal et al, 2008). However, there remains uncertainty with regard to whether low-risk FN in adult cancer patients is best managed in-hospital or in an outpatient setting. Some of this uncertainty is related to questions as to whether there are differences in the safety or efficacy of the different management strategies. Thus far, no study has quantitatively synthesised the evidence comparing outpatient $v s$ in-patient management. Additional uncertainty is related to health-related quality of life and preferences of patients

*Correspondence: Dr L Sung; E-mail: lillian.sung@sickkids.ca Received 22 November 2010; revised 28 February 2011; accepted 2 March 20I I; published online 5 April 20I I in the context of FN (Brown et al, 2001; Havrilesky et al, 2009). In contrast, there is a strong body of evidence published in the literature suggesting that costs of in-hospital treatment are greater than the costs of ambulatory care for FN (Bennett and Calhoun, 2007; Liou et al, 2007). A major limitation of current data is whether higher costs of in-patient care can be justified on the basis of safety and efficacy issues, or patients' preferences. Clarifying this issue may have major resource implications given the relative frequency and costs associated with admission to hospital with FN.

Therefore, the objective of this study was to construct a cost-utility model to determine the optimal treatment strategy for low-risk FN in adults with cancer. In this study, costs and effectiveness (measured as quality-adjusted FN episodes (QAFNE)) of four different treatment strategies for low-risk FN were examined. These included entire in-patient management, treatment at home after an initial observation in hospital, entire outpatient management with IV antibiotics and entire outpatient management with oral antibiotics.

\section{MATERIALS AND METHODS}

\section{Overview}

Adult cancer patients with low-risk FN were entered into a decision-analytic model as a hypothetical cohort. The decision tree 
model encompassed one episode of FN. A simple decision tree model was chosen rather than a state-transition model (Markov model) for two reasons: (1) the concept of changing transition probabilities between different health states did not apply to our clinical scenario (acute health state); (2) there are no data in the literature indicating that long-term implications of a single low-risk episode differ between strategies (Rubenstein et al, 1993; Malik et al, 1995; Hidalgo et al, 1999; Minotti et al, 1999; Rapoport et al, 1999; Innes et al, 2003; Sebban et al, 2008). Death was not included in the model, because this is a very unusual event in low-risk FN as described in the literature. The rare cases of death reported from randomised controlled trials (RCTs) appear to be randomly distributed between in- and outpatient settings (Malik et al, 1995; Hidalgo et al, 1999; Rapoport et al, 1999; Innes et al, 2003). Furthermore, there has not been a single case of death related to infection in any observational study of outpatient management reported within the last 20 years (Talcott et al, 1994; Escalante et al, 2004; Chamilos et al, 2005; Klastersky et al, 2006; Rolston et al, 2006). Thus, inclusion of death would not substantially contribute to preferences for any one strategy. Consequently, effectiveness was measured as QAFNE rather than quality-adjusted life years (QALYs), which is the preferred outcome measure in state-transition models (Siegel et al, 1996).

\section{Decision-analytical model}

A decision-analytic model was constructed using TreeAge Pro 2009 and examined four strategies: (1) entire treatment in hospital with IV antibiotics (HospIV); (2) early discharge consisting of $48 \mathrm{~h}$ in-patient observation with IV antibiotics, followed by oral outpatient treatment (EarlyDC); (3) entire outpatient management with IV antibiotics (HomeIV); and (4) entire outpatient management with oral antibiotics (HomePO). Piperacillin/tazobactam plus tobramycin was chosen as first-line IV antibiotics, whereas oral treatment consisted of ciprofloxacin plus amoxicillin/clavulanate (Hughes et al, 2002). Consistent with the published literature, average treatment duration was defined as 6 days for all four strategies (Rubenstein et al, 1993; Rapoport et al, 1999; Innes et al, 2003; Sebban et al, 2008). Outcome measures were QAFNE, costs (in Canadian dollars) and incremental cost-effectiveness ratios (ICER). The structure of the decision tree and its pathways are illustrated in Figure 1. Further details of the decision-analytic model are outlined in Supplementary data A.

\section{Probabilities}

A systematic review was conducted to obtain the best available evidence with regard to outpatient management of FN (only RCTs were considered) (Teuffel et al, 2011). An electronic search of OVID MEDLINE from 1950 to February 2010, EMBASE from 1980 to February 2010 and The Cochrane Central Register of Controlled Trials (first quarter of 2010) was performed. Conference Proceedings from 2007 to 2010 were retrieved from ISI Web of Science and SCOPUS. From 1448 reviewed articles, seven studies met pre-defined inclusion criteria (Rubenstein et al, 1993; Malik et al, 1995; Hidalgo et al, 1999; Minotti et al, 1999; Rapoport et al, 1999; Innes et al, 2003; Sebban et al, 2008). 'Low risk' was defined as per study protocol.(Teuffel et al, 2011) The mean rates of treatment failure and hospital readmission were extracted from the seven studies stated above (Table 1). The time

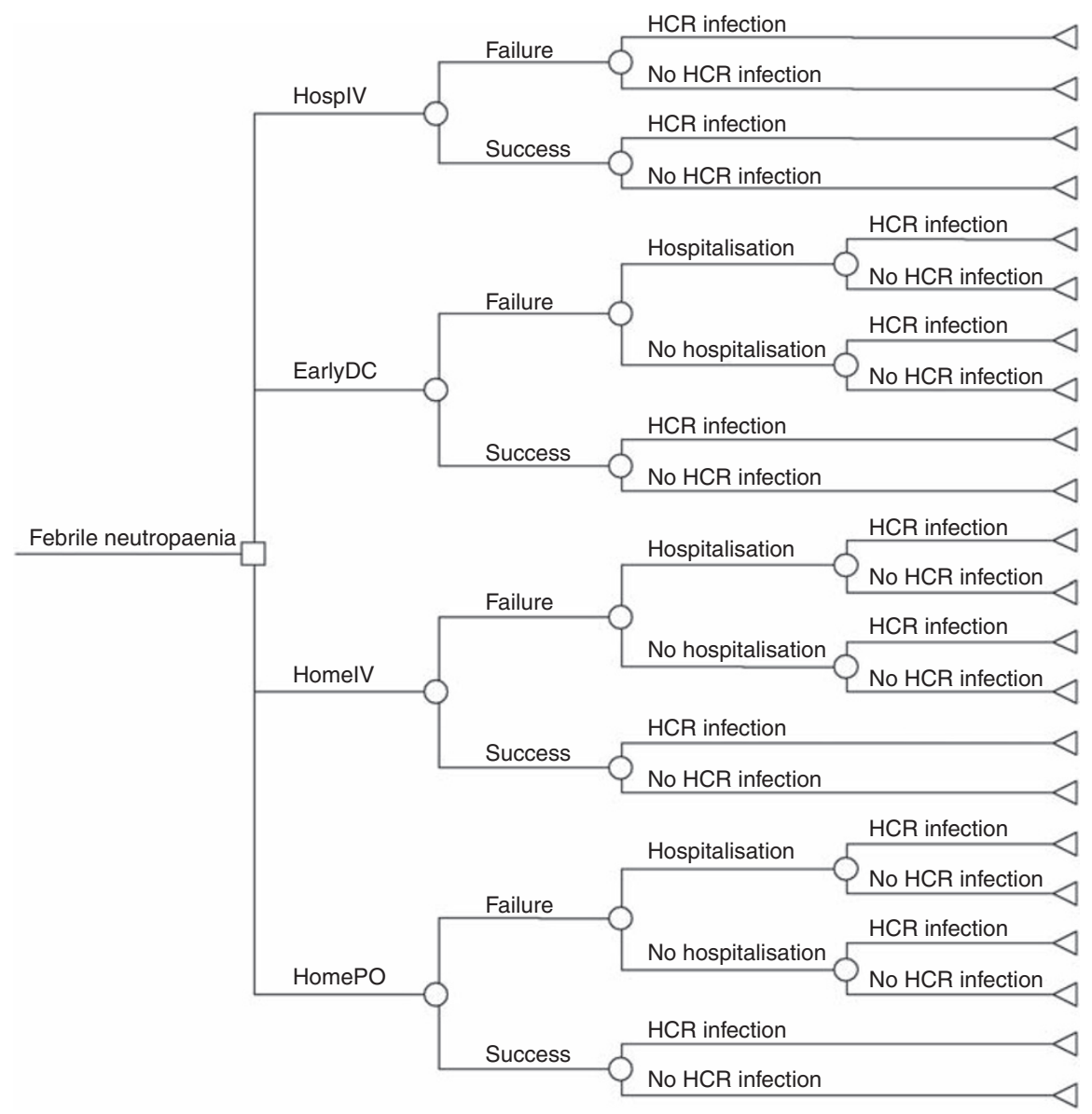

Figure I Clinical decision model to compare different treatment strategies for low-risk febrile neutropaenia in adult cancer patients; four treatment strategies are evaluated: (I) entire HosplV; (2) EarlyDC; (3) HomelV; and (4) HomePO. HCR indicates health-care-related infection. 
Table I Model parameter and distributions

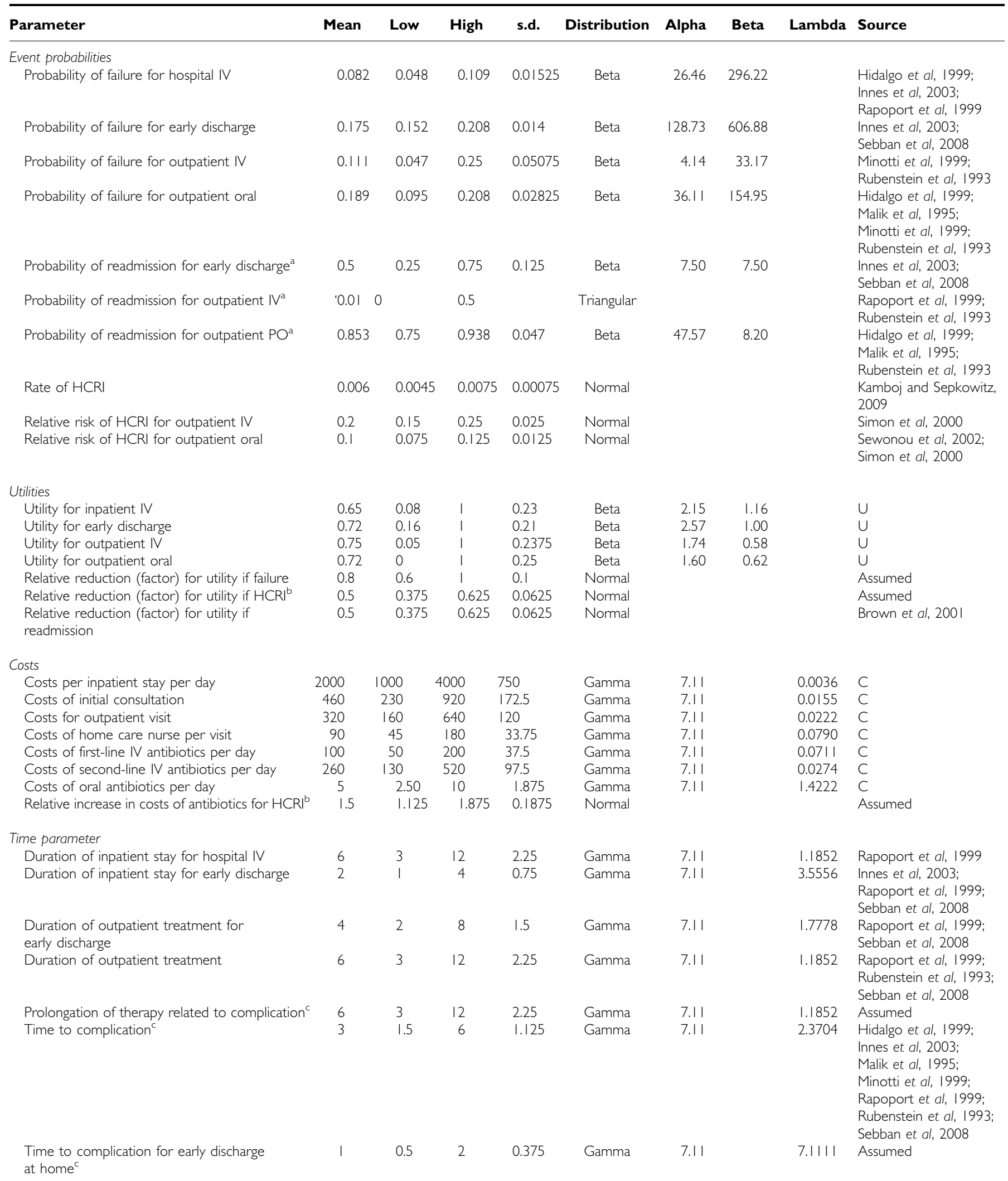

C: costs were obtained from local finance offices and the Department of Pharmacy at Princess Margaret Hospital in Toronto, Ontario/Canada. U: utilities (visual analogue scale scores, converted into standard gamble utilities) were derived from 77 adult cancer patients at Princess Margaret Hospital in Toronto, Ontario/Canada. ${ }^{2} \mathrm{Conditional} \mathrm{on} \mathrm{failure} \mathrm{of}$ therapy. ${ }^{b}$ Healthcare-related infection. ${ }^{~}$ Complication = failure, readmission, healthcare-related infection. Note: the probability of readmission for outpatient IV was $0 \%$ based on two published RCTs; however, to apply a reasonable distribution to this variable ( $\rightarrow$ triangular), a peak estimate of $1 \%(0.01)$ was chosen. 
frame for events as reported by included studies encompassed 30 days. The range of possible outcomes was estimated from studies describing the most extreme results (Table 1). The probability of health-care-related infections was obtained from several observational studies (Simon et al, 2000, 2008; Kamboj and Sepkowitz, 2009).

\section{Utilities}

Health utilities reflect quantitative assessments of the strength of individual preferences for health states when measured under conditions of uncertainty (VonNeumann and Morgenstern, 1953; Torrance, 1987). We elicited preferences from 77 patients receiving active treatment for cancer at Princess Margaret Hospital $(\mathrm{PMH}$, Toronto, Canada), with regard to the four different treatment strategies. A current or previous episode of FN was not mandatory for inclusion. Hypothetical scenarios were presented (Supplementary data B) and a visual analogue scale (VAS) was used to measure patients' preferences for the four different health states (strategies) (Patrick et al, 1973; Drummond et al, 2005). Respondents were asked to mark their preferences on a horizontal $10-\mathrm{cm}$ line anchored at the left end by the worst possible health or death (score of 0 ) and at the right end by perfect health (score of 1). Visual analogue scale scores are not usually considered utilities (because they are measured under conditions of certainty), but they are related to standard gamble utilities in a nonlinear manner and can be transformed to the latter using a power function (Torrance et al, 2001). The following conversion algorithm was used in this study for deriving a standard gamble score (Table 1): $1-(1-\mathrm{VAS})^{1.61}$ (Torrance, 1976; Torrance et al, 2001). Utility deductions for treatment failure, readmission and health-carerelated infection were applied (Table 1) to each of the four treatment options to arrive at final utilities for all 22 possible health states (Figure 1). Effectiveness (QAFNE) was derived from the weighted average products of health state probabilities and health state utilities for each pathway.

\section{Costs}

The analysis adopted the health-care payer's perspective in Canada and included all relevant direct health costs. Cost factors associated with hospitalisation, initial consultation, outpatient

Table 2 Base-case analysis

\begin{tabular}{lcccc}
\hline & HomePO & HomelV & EarlyDC & HosplV \\
\hline Cost (mean) & $\$ 3470$ & $\$ 4183$ & $\$ 6115$ & $\$ 13557$ \\
Cost $(95 \% \mathrm{Cl})$ & $\$ 1669-6564$ & $\$ 2001-7616$ & $\$ 2471-12394$ & $\$ 4592-30000$ \\
IncrC (mean) & - & $\$ 713$ & $\$ 1932$ & $\$ 9374$ \\
Eff (mean) & 0.65 & 0.72 & 0.66 & 0.62 \\
Eff $(95 \% \mathrm{Cl})$ & $0.13-0.91$ & $0.18-0.98$ & $0.22-0.92$ & $0.15-0.94$ \\
IncrEff (mean) & - & 0.07 & -0.06 & -0.10 \\
C/E (mean) & $\$ 5338$ & $\$ 5810$ & $\$ 9265$ & $\$ 21866$ \\
ICER & - & $\$ 10186$ & Dominated $^{\mathrm{a}}$ & Dominated $^{\mathrm{a}}$ \\
\hline
\end{tabular}

Abbreviations: $\mathrm{Cl}=$ confidence interval; $\mathrm{C} / \mathrm{E}=$ cost-effectiveness ratio; EarlyDC = treatment at home after an initial observation in hospital; Eff, effectiveness; Effectiveness = quality-adjusted febrile neutropaenia episode (rounded to 2 decimals); HomelV = outpatient management with intravenous antibiotics; Home$\mathrm{PO}=$ outpatient management with oral antibiotics; HosplV =entire in-patient management; IncrC, incremental cost; IncrEff, incremental effectiveness; ICER, incremental cost-effectiveness ratio. ${ }^{a}$ Dominated refers to the finding that this strategy is dominated (e.g. both less effective and more costly than other strategies). Note: All costs are given in Canadian dollars. Options are ordered by increasing costs. Option I (HomePO) is the baseline reference to calculate incremental costs and effectiveness for option 2 (HomelV). As option 2 is more effective than option I, the former one is used as new baseline reference to calculate incremental costs (and effectiveness) for options 3 (EarlyDC) and 4 (HosplV). As options 3 and 4 are less effective and more expensive than option 2, they are both dominated by option 2 . visits, home care nursing and medications were obtained from the following sources: (1) Ontario Health Insurance Schedule of Benefits and Fees; (2) local finance offices at PMH (hospital fees/charges and home care nurse visits); and (3) the Department of Pharmacy at $\mathrm{PMH}$ (drug costs). The following range was applied to the probabilistic analyses: lower limit $=$ baseline estimate $\times 0.5$ and upper limit $=$ baseline estimate $\times 2$ (Table 1$)$. Detailed information about cost factors are given in Supplementary data C. All costs are quoted in 2009 Canadian dollars. As all outcomes occurred within one episode of FN, no discounting was applied.

\section{Probabilistic sensitivity analysis}

A probabilistic sensitivity analysis was performed to address the joint uncertainty of all model parameters simultaneously and to provide a more accurate estimate of the average ICER. We performed 10000 second-order Monte Carlo simulations and reported results in two ways. First, we reported mean health outcomes and costs with $95 \%$ confidence intervals, that is, the range covered by $95 \%$ of our simulations. Second, we graphed results on a cost-effectiveness acceptability curve, which starts from the assumption that cost-effectiveness ratios are considered attractive if the estimates are less than the amount society would be willing to pay for an additional unit gain in health. The curve was generated that used net benefits to graph the changing percentage of simulations for which each comparator is cost effective relative to all other strategies.

As a secondary analysis, all variables were tested in a one-way (plausible range) deterministic sensitivity analysis.

For all analyses, the willingness-to-pay (WTP) threshold was set at $\$ 4000$ per QAFNE, with a range testing from $\$ 0$ to $\$ 20000$ for

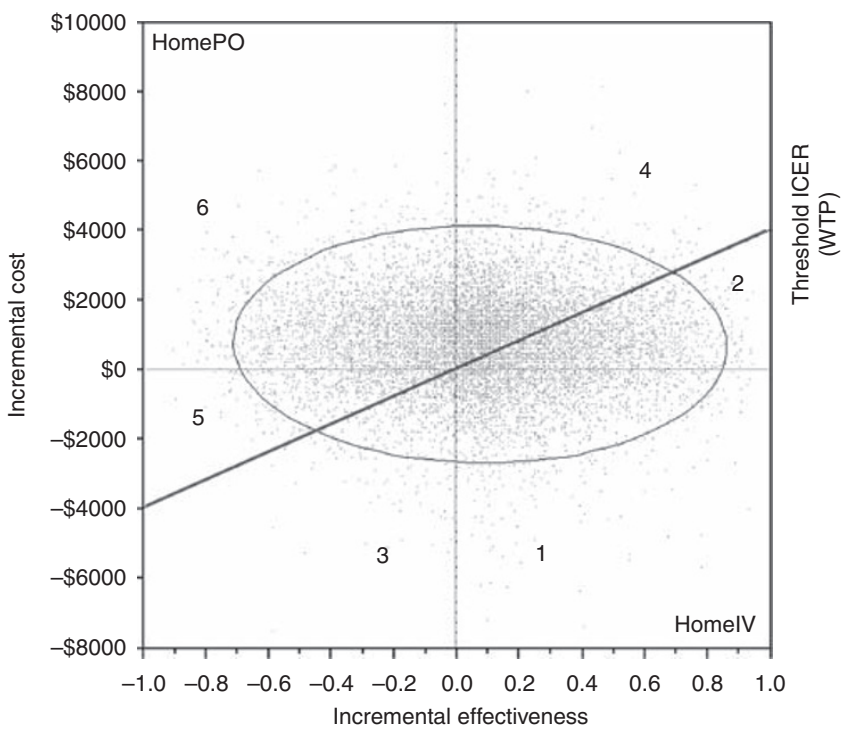

Figure 2 The ICE scatter plot includes a single set of points representing pairs of incremental cost and effectiveness values from the simulation results $(n=10000)$ relative to a baseline (oral treatment at home; HomePO). The comparator in this scatter plot is IV treatment at home (HomelV). The slope intersecting the $y$ axis at $\$ 4000$ displays the WTP threshold. In addition to the WTP line, a 95\% confidence ellipse is drawn in the ICE scatter plot. The graph can be divided into several distinct regions: (I) HomelV dominates HomePO (17\%); (2) HomelV is more costly and effective, and its ICER is less than or equal to the WTP, so it is cost effective (19\%): (3) HomePO is more costly and effective, but its ICER is greater than the WTP, so HomelV is optimal (4\%); (4) HomelV is more costly and effective, but its ICER is greater than the WTP, so HomePO is optimal (26\%); (5) HomePO is more costly and effective, and its ICER is less than or equal to the WTP, so its optimal (6\%); and (6) HomePO dominates HomelV (28\%). 


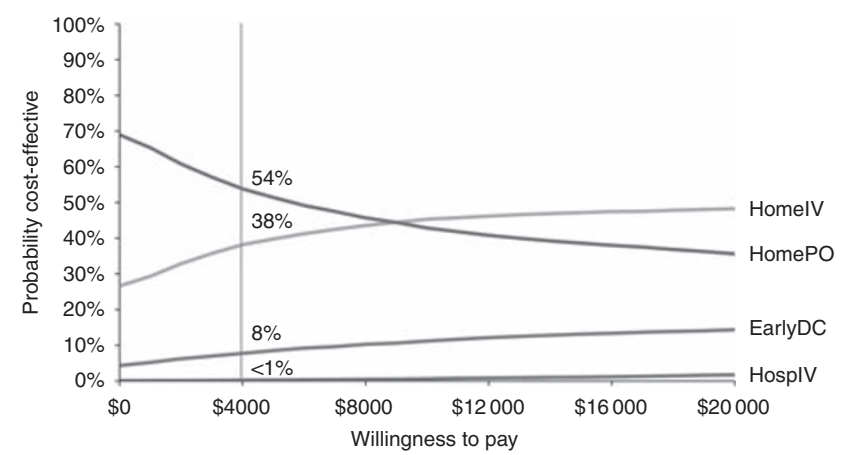

Figure 3 This chart presents the cost-effectiveness acceptability curve for the base-case analysis. The curves represent the proportion of simulations in which oral outpatient therapy and IV outpatient therapy, respectively, were the cost-effective option at various WTP thresholds. For example, at a WTP threshold of $\$ 4000$ per quality-adjusted febrile neutropaenia episode (vertical axis), oral therapy was cost effective in 54\% of the simulations.

the acceptability curve. We assumed that one episode of FN did not exceed 1 month (time frame of event probabilities $=30$ days). Hence, $\$ 4000$ per QAFNE was used as baseline value as this approximates a WTP threshold of $\$ 50000$ per QALY, a threshold commonly used in health economic evaluations (Shiroiwa et al, 2010).

\section{RESULTS}

\section{Base-case analysis}

Table 2 lists costs per episode and effectiveness of the four strategies under study. The model predicted that the average costs for oral outpatient management for adult cancer patients with lowrisk FN would be lower than the costs for IV outpatient management, for the early discharge strategy and for in-hospital management. Considering quality of life, oral outpatient management yielded an average QAFNE of 0.65. This was inferior to outpatient IV and the early discharge option, but superior to the in-hospital strategy. Thus, our cost-utility analysis revealed that the oral outpatient strategy was cost saving, but less effective than its outpatient IV counterpart. The corresponding ICER was \$10 186 per QAFNE. Both the early discharge strategy and in-hospital therapy were less cost effective than either outpatient strategies. Incremental cost-effectiveness ratios could not be calculated for the latter two treatment approaches as they were dominated strategies (Table 2). An ICE scatter plot comparing the two most costeffective strategies (baseline $=$ outpatient oral $v s$ comparator $=$ outpatient IV) is shown in Figure 2.

\section{Acceptability curve}

At a WTP threshold of $\$ 4000$ per QAFNE, oral outpatient treatment was cost effective in $54 \%$ of simulations, whereas outpatient IV was cost effective in $38 \%$ of the simulations. The early discharge strategy was cost effective in $8 \%$ and the traditional in-patient management was cost effective in less than $1 \%$ of the simulations. The results of the cost-effectiveness acceptability curve are shown in Figure 3.

\section{One-way deterministic sensitivity analysis}

When tested for costs within the plausible range in a one-way sensitivity analysis, the model was sensitive to costs for in-patient stay per day (threshold $>\$ 2455$ ), outpatient visit (threshold $<\$ 191$ ) and home care nurse per visit (threshold $<\$ 49)$. The model was also sensitive within the plausible range to the following variables: duration of outpatient treatment (threshold $<4.9$ days); prolongation of therapy owing to complications (threshold $>7.6$ days); utility for outpatient IV (threshold $>0.851$ ); and utility for outpatient oral therapy (threshold <0.611). Beyond certain thresholds, dominance (less expensive and more effective) changed from the outpatient oral to the outpatient IV strategy. Importantly, there was not a single constellation when EarlyDC or HospIV became superior to the other two strategies.

\section{DISCUSSION}

This study suggests that outpatient strategies are the preferred approach to manage adult cancer patients with low-risk FN. Both outpatient oral and outpatient IV therapy are more cost effective than EarlyDC or entire in-patient management. This is, to our knowledge, the first model that addresses the question as to whether FN within the low-risk patient population is best managed in hospital or in an outpatient setting.

The probabilistic analysis in this study showed that in-patient care for FN was the least cost-effective strategy even when tested over a wide range of inputs/plausible values (in-patient care was cost effective in less than $1 \%$ of all Monte Carlo simulations). A likely explanation for the inferiority of the in-patient strategy in our model is its associated high costs. We found that the mean direct health costs were four-fold greater for in-patients as compared with oral outpatient management (Bennett and Calhoun, 2007). The economic burden for in-patient care in our study was higher than that in another recently published Canadian study (Lathia et al, 2010). On the contrary, one in-patient episode of FN in our model was, on average, less expensive as compared with recent cost estimates from the United States (Elting et al, 2008). We suggest that our single institution's estimates - given the plausible range/distribution used for the probabilistic analyses permit broad applicability to other settings (Liou et al, 2007). In addition to higher costs, in-hospital care also seems to be associated with decreased health-related quality of life, at least at the aggregate level.

In contrast, our model was unable to consistently show the superiority of one of the two ambulatory strategies, namely outpatient oral and outpatient IV treatment. However, it appears reasonable that these two outpatient approaches present similar cost-effectiveness ratios. Moreover, having two comparable approaches in terms of cost effectiveness might allow patients to play a more active role in decision making in future, particularly if probabilities of treatment failure are similar between strategies, although other concerns such as feasibility may drive this decision.

Our cost-utility analysis has a number of important limitations. First, only limited data were available to estimate the base values in the model, leading to uncertainty with regard to the precision of the included event probabilities. To minimise this limitation, data obtained from a formal systematic review were applied across a wide range of values in our analyses. Second, we used VAS scores to derive health-related quality of life estimates for adult cancer patients with FN. To adjust for this limitation, we converted the VAS scores into standard gamble values by applying an equation developed by Torrance in the mid-1970s (Torrance, 1976; Torrance et al, 2001). Although not all studies have shown this technique to be valid, the credibility of our calculated standard gamble values has been supported by a recent study using the EQ-5D tool to elicit patients' preferences for in-patient management of FN (Torrance et al, 2001; Lathia, 2008). In the cited study, a mean health utility of 0.64 was calculated, which is almost identical to our converted utility estimate for patients treated entirely in hospital (mean standard gamble value 0.65) (Lathia, 2008). Third, the base-case analysis was performed using a hypothetical cohort. This might be advantageous in terms of external validity; however, the data are not easily applicable at the individual level owing to both covert 
and overt (e.g. preference) variability within this cohort. Fourth, the ICER of $\$ 10186$ reported in our study together with the WTP threshold set at $\$ 4000$ are difficult to interpret as FN episodes do not happen 12 times per year (to remember, $\$ 4000$ per QAFNE was used as baseline value as this approximates a WTP threshold of $\$ 50000$ per QALY). Determining a reasonable ICER threshold for relatively short-term events remains contentious (Birch and Gafni, 2006). However, we suggest that an ICER of approximately $\$ 10000$ related to a transient episode that is unlikely to occur more than 2-3 times per year might be acceptable, at least for some countries. Nevertheless, it is difficult to define an acceptable ICER threshold for this decision-analytic model owing to the economic complexity associated with cancer treatment, including both the antineoplastic treatment itself and side effects other than FN.

Our study is important as it is the first quantitative attempt to compare different treatment strategies for low-risk FN in adult cancer patients. However, there are several important questions related to feasibility and individual preferences that need to be addressed in future research. First, are outpatient strategies feasible in settings such as lower-income countries and rural areas of high-income countries? The external validity of safe outpatient management has had limited evaluation outside the carefully controlled setting of RCTs. Second, further research is necessary to address the issue of preferences and medical decision-making. If efficacy and safety do not substantially differ between strategies, patients' preferences should impact on choosing the appropriate treatment setting and the way of drug administration ('individualised patient care'). However, obtaining and interpreting patients' preferences for acute or non-fatal conditions can be problematic (Bala and Zarkin, 2000). 'Bed-side' tools that can be used to support the decision-making process would be highly desirable.

To conclude, our model suggests in adult cancer patients with an episode of low-risk FN, outpatient strategies are preferable to inhospital treatment with IV antibiotics. Future research should focus on the feasibility of outpatient approaches in different settings, mechanisms to facilitate such an approach and the determination of patients' preferences.

\section{ACKNOWLEDGEMENTS}

A bursary from the Swiss Cancer League supports OT. SA is a Research Scientist of the Canadian Cancer Society. LS is supported by a New Investigator Award from the Canadian Institutes of Health Research. JB acknowledges funding from the Canadian Institute of Health Research, CIHR (Grant no. 84392).

Supplementary Information accompanies the paper on British Journal of Cancer website (http://www.nature.com/bjc)

\section{REFERENCES}

Bala MV, Zarkin GA (2000) Are QALYs an appropriate measure for valuing morbidity in acute diseases? Health Econ 9: 177-180

Bennett CL, Calhoun EA (2007) Evaluating the total costs of chemotherapyinduced febrile neutropenia: results from a pilot study with community oncology cancer patients. Oncologist 12: $478-483$

Birch S, Gafni A (2006) Information created to evade reality (ICER): things we should not look to for answers. Pharmacoeconomics 24: 1121-1131

Brown RE, Hutton J, Burrell A (2001) Cost effectiveness of treatment options in advanced breast cancer in the UK. Pharmacoeconomics 19: $1091-1102$

Chamilos G, Bamias A, Efstathiou E, Zorzou PM, Kastritis E, Kostis E, Papadimitriou C, Dimopoulos MA (2005) Outpatient treatment of lowrisk neutropenic fever in cancer patients using oral moxifloxacin. Cancer 103: $2629-2635$

Drummond MF, Sculpher MJ, Torrance GW, O'Brien BJ, Stoddart GL (2005) Methods for the Economic Evaluation of Health Care Programmes, 3rd edn. Oxford University Press: New York

Elting LS, Lu C, Escalante CP, Giordano SH, Trent JC, Cooksley C, Avritscher EB, Shih YC, Ensor J, Bekele BN, Gralla RJ, Talcott JA, Rolston $\mathrm{K}$ (2008) Outcomes and cost of outpatient or inpatient management of 712 patients with febrile neutropenia. J Clin Oncol 26: 606-611

Escalante CP, Weiser MA, Manzullo E, Benjamin R, Rivera E, Lam T, Ho V, Valdres R, Lee EL, Badrina N, Fernandez S, DeJesus Y, Rolston K (2004) Outcomes of treatment pathways in outpatient treatment of low risk febrile neutropenic cancer patients. Support Care Cancer 12: 657-662

Havrilesky LJ, Broadwater G, Davis DM, Nolte KC, Barnett JC, Myers ER, Kulasingam S (2009) Determination of quality of life-related utilities for health states relevant to ovarian cancer diagnosis and treatment. Gynecol Oncol 113: $216-220$

Hidalgo M, Hornedo J, Lumbreras C, Trigo JM, Colomer R, Perea S, Gemez C, Ruiz A, Garcia-Carbonero R, Cortes-Funes H (1999) Outpatient therapy with oral ofloxacin for patients with low risk neutropenia and fever: a prospective, randomized clinical trial. Cancer 85: 213-219

Hughes WT, Armstrong D, Bodey GP, Bow EJ, Brown AE, Calandra T, Feld R, Pizzo PA, Rolston KV, Shenep JL, Young LS (2002) 2002 guidelines for the use of antimicrobial agents in neutropenic patients with cancer. Clin Infect Dis 34: 730-751

Innes HE, Smith DB, O’Reilly SM, Clark PI, Kelly V, Marshall E (2003) Oral antibiotics with early hospital discharge compared with in-patient intravenous antibiotics for low-risk febrile neutropenia in patients with cancer: a prospective randomised controlled single centre study (see comment). $\mathrm{Br}$ J Cancer 89: $43-49$
Kamboj M, Sepkowitz KA (2009) Nosocomial infections in patients with cancer. Lancet Oncol 10: 589-597

Klastersky J, Paesmans M, Georgala A, Muanza F, Plehiers B, Dubreucq L, Lalami Y, Aoun M, Barette M (2006) Outpatient oral antibiotics for febrile neutropenic cancer patients using a score predictive for complications. J Clin Oncol 24: 4129-4134

Klastersky J, Paesmans M, Rubenstein EB, Boyer M, Elting L, Feld R, Gallagher J, Herrstedt J, Rapoport B, Rolston K, Talcott J (2000) The multinational association for supportive care in cancer risk index: a multinational scoring system for identifying low-risk febrile neutropenic cancer patients. J Clin Oncol 18: $3038-3051$

Lathia N (2008) Evaluation of direct medical costs, lost productivity, health utility and quality of life in patients hospitalized for febrile neutropenia. Master of Science University of Toronto: Toronto, Canada

Lathia N, Mittmann N, DeAngelis C, Knowles S, Cheung M, Piliotis E, Shear N, Walker S (2010) Evaluation of direct medical costs of hospitalization for febrile neutropenia. Cancer 116: $742-748$

Liou SY, Stephens JM, Carpiuc KT, Feng W, Botteman MF, Hay JW (2007) Economic burden of haematological adverse effects in cancer patients: a systematic review. Clin Drug Invest 27: $381-396$

Malik IA, Khan WA, Karim M, Aziz Z, Khan MA (1995) Feasibility of outpatient management of fever in cancer patients with low-risk neutropenia: results of a prospective randomized trial [see comment]. Am J Med 98: 224-231

Minotti V, Gentile G, Bucaneve G, Iori AP, Micozzi A, Cavicchi F, Barbabietola G, Landonio G, Menichetti F, Martino P, Del Favero A (1999) Domiciliary treatment of febrile episodes in cancer patients: a prospective randomized trial comparing oral versus parenteral empirical antibiotic treatment. Support Care Cancer 7: 134-139

Patrick DL, Bush JW, Chen MM (1973) Methods for measuring levels of well-being for a health status index. Health Serv Res 8: 228-245

Rapoport BL, Sussmann O, Herrera MV, Schlaeffer F, Otero JC, Pavlovsky S, Iglesias L, Stein G, Charnas R, Heitlinger E, Handschin J (1999) Ceftriaxone plus once daily aminoglycoside with filgrastim for treatment of febrile neutropenia: early hospital discharge $v s$ standard inpatient care. Chemotherapy 45: $466-476$

Renwick W, Pettengell R, Green M (2009) Use of filgrastim and pegfilgrastim to support delivery of chemotherapy: twenty years of clinical experience. BioDrugs 23: $175-186$

Rolston KVI, Manzullo EF, Elting LS, Frisbee-Hume SE, McMahon L, Theriault RL, Patel S, Benjamin RS (2006) Once daily, oral, outpatient quinolone monotherapy for low-risk cancer patients with fever and 
neutropenia: a pilot study of 40 patients based on validated riskprediction rules. Cancer 106: 2489-2494

Rubenstein EB, Rolston K, Benjamin RS, Loewy J, Escalante C, Manzullo E, Hughes P, Moreland B, Fender A, Kennedy K (1993) Outpatient treatment of febrile episodes in low-risk neutropenic patients with cancer. Cancer 71: $3640-3646$

Sebban C, Dussart S, Fuhrmann C, Ghesquieres H, Rodrigues I, Geoffrois L, Devaux Y, Lancry L, Chvetzoff G, Bachelot T, Chelghoum M, Biron P (2008) Oral moxifloxacin or intravenous ceftriaxone for the treatment of low-risk neutropenic fever in cancer patients suitable for early hospital discharge. Support Care Cancer 16: 1017-1023

Segal BH, Freifeld AG, Baden LR, Brown AE, Casper C, Dubberke E, Gelfand M, Greene JN, Ison MG, Ito JI, Karp JE, Kaul DR, King E, Mackler E, Marcucci G, Montoya JG, Engemann AM, Rolston K, The AS (2008) Prevention and treatment of cancer-related infections. J Natl Compr Canc Netw 6: $122-174$

Shiroiwa T, Sung YK, Fukuda T, Lang HC, Bae SC, Tsutani K (2010) International survey on willingness-to-pay (WTP) for one additional QALY gained: what is the threshold of cost effectiveness? Health Econ 19: $422-437$

Siegel JE, Weinstein MC, Russell LB, Gold MR (1996) Recommendations for reporting cost-effectiveness analyses. Panel on cost-effectiveness in health and medicine. JAMA 276: 1339-1341

Simon A, Ammann RA, Bode U, Fleischhack G, Wenchel HM, Schwamborn D, Gravou C, Schlegel PG, Rutkowski S, Dannenberg C, Korholz D, Laws $\mathrm{HJ}$, Kramer $\mathrm{MH}$ (2008) Healthcare-associated infections in pediatric cancer patients: results of a prospective surveillance study from university hospitals in Germany and Switzerland. BMC Infect Dis 8: 70

Simon A, Fleischhack G, Hasan C, Bode U, Engelhart S, Kramer MH (2000) Surveillance for nosocomial and central line-related infections among pediatric hematology-oncology patients. Infect Control Hosp Epidemiol 21: $592-596$

Talcott JA, Whalen A, Clark J, Rieker PP, Finberg R (1994) Home antibiotic therapy for low-risk cancer patients with fever and neutropenia: a pilot study of 30 patients based on a validated prediction rule. J Clin Oncol 12: $107-114$

Tamura K (2005) Clinical guidelines for the management of neutropenic patients with unexplained fever in Japan: validation by the Japan Febrile Neutropenia Study Group. Int J Antimicrob Agents 26(Suppl 2): S123-S127; discussion S133-40

Teuffel O, Ethier MC, Alibhai SMH, Beyene J, Sung L (2011) Outpatient management of cancer patients with febrile neutropenia: a systematic review and meta-analysis. Ann Oncol 22; E-pub ahead of print 1 March 2011

Torrance GW (1976) Social preferences for health states: an empirical evaluation of three measurement techniques. Socioecon Plann Sci 10: $129-136$

Torrance GW (1987) Utility approach to measuring health-related quality of life. J Chronic Dis 40: 593-603

Torrance GW, Feeny D, Furlong W (2001) Visual analog scales: do they have a role in the measurement of preferences for health states? Med Decis Making 21: 329-334

VonNeumann J, Morgenstern O (1953) Theory of Games and Economic Behaviour, 3rd edn. Princeton: New York 